\title{
Reciprocal Relation between Spin Peltier and Spin Seebeck Effects ${ }^{+}$
}

\author{
Alessandro Sola ${ }^{1, *}$, Vittorio Basso ${ }^{1}$, Michaela Kuepferling ${ }^{1}$, Carsten Dubs ${ }^{2}$ and \\ Massimo Pasquale ${ }^{1}$ \\ 1 Istituto Nazionale di Ricerca Metrologica, Strada delle Cacce 91, 10135, Turin, Italy \\ 2 INNOVENT e.V., Technologieentwicklung, Prussingstrasse. 27B, 07745 Jena, Germany \\ * Correspondence: a.sola@inrim.it \\ † Presented at the 37th International Symposium on Dynamical Properties of Solids (DyProSo 2019), Ferrara, \\ Italy, 8-12 September 2019.
}

Published: 5 September 2019

In recent times, the interaction between magnetization and heat currents in a magnetic material has gained a renewed interest thanks to the observation of the spin Seebeck effect (SSE) [1,2]. The SSE is the spin counterpart of the Seebeck effect that corresponds to the generation of a pure magnetization current in a magnetic insulator as consequence of a thermal gradient. This is electrically detected by means of the inverse spin Hall effect [3], that rises in a high spin orbit coupling heavy metal deposited on the magnetic insulator. Equally to the ordinary thermoelectricity, the SSE has its reciprocal effect that is the spin Peltier effect [4,5]. In this work we provide an experimental proof of the reciprocal relations between SSE and SPE [6,7] in a single bulk sample of yttrium iron garnet (YIG) covered by a platinum thin film. For both the SSE and the SPE experiments, we employ a measurement system designed for the detection of heat currents exchanged between the thermal reservoirs and the sample under test. The sample-specific value for the characteristics of both effects measured on the present YIG/Pt bilayer is $(6.2 \pm 0.4) \times 10^{-3} \mathrm{KA}^{-1}$ at room temperature, that corresponds to the analogous for spins of the Thomson relation between thermoelectric effects.

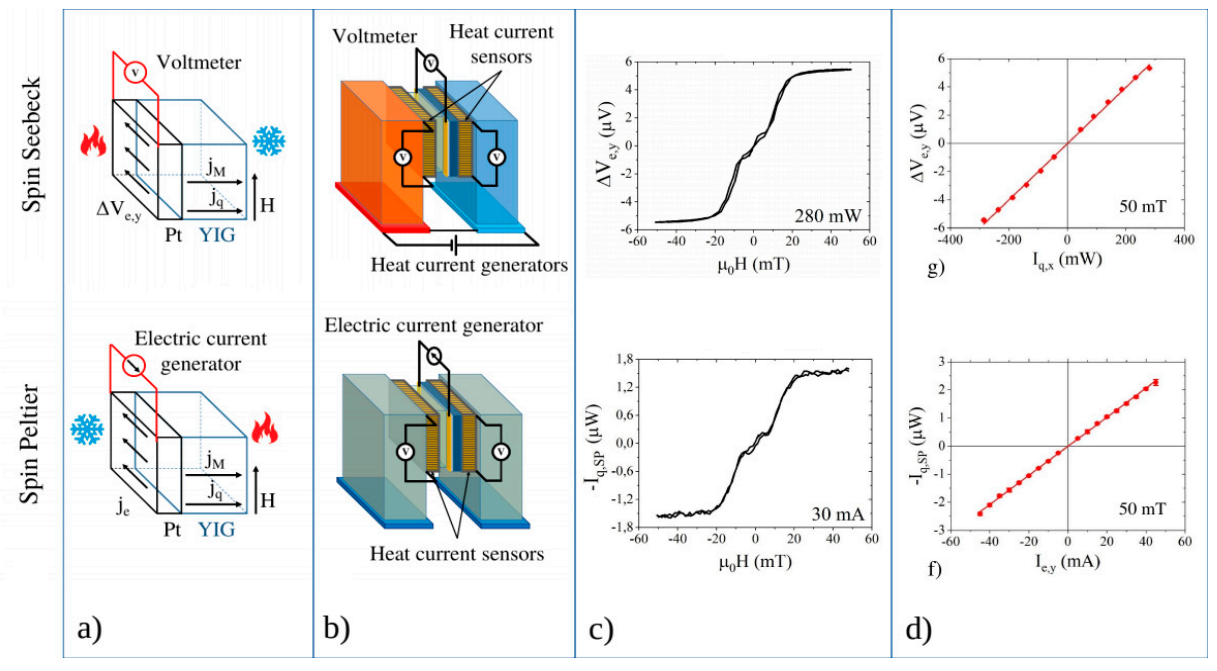

Figure 1. SSE and SPE heat-current-based measurements. (a) Schemes of the YIG-Pt device. (b) Sketches of the experimental setups. (c) Examples of SSE and SPE hysteresis loops. (d) Results at magnetic saturation of the SSE voltage as function of the heat current and SPE heat current as function of the electric current. 


\section{References}

1. Uchida, K.; Adachi, H.; Ota, T.; Nakayama, H.; Maekawa, S.; Saitoh, E. Observation of longitudinal spinSeebeck effect in magnetic insulators. Appl. Phys. Lett. 2010, 97, 172505.

2. Bauer, G.E.W.; Saitoh, E.; van Wees, B.J. Spin caloritronics. Nat. Mater. 2012, 391, 11.

3. Saitoh, E.; Ueda, M.; Miyajima, H.; Tatara, G. Conversion of spin current into charge current at room temperature: Inverse spin-Hall effect. Appl. Phys. Lett. 2006, 88, 182509.

4. Flipse, J.; Dejene, F.; Wagenaar, D.; Bauer, G.E.W.; Youssef, J.B.; van Wees, B. Observation of the spin Peltier effect for magnetic insulators. Phys. Rev. Lett. 2014, 113, 027601.

5. Daimon, S.; Uchida, K.; Iguchi, R.; Hioki, T.; Saitoh, E. Thermographic measurements of the spin Peltier effect in metal/yttrium-iron-garnet junction systems. Phys. Rev. B 2017, 96, 024424.

6. Basso, V.; Kuepferling, M.; Sola, A.; Ansalone, P.; Pasquale, M. The spin Seebeck and spin Peltier reciprocal relation. IEEE Magn. Lett. 2018, 9, 1-4.

7. Sola, A.; Basso, V.; Kuepferling, M.; Dubs, C.; Pasquale, M. Experimental proof of the reciprocal relation between spin Peltier and spin Seebeck effects in a bulk YIG/Pt bilayer. Sci. Rep. 2019, 9, 204.

(C) 2019 by the authors. Licensee MDPI, Basel, Switzerland. This article is an open access article distributed under the terms and conditions of the Creative Commons Attribution (CC BY) license (http://creativecommons.org/licenses/by/4.0/). 